\title{
ThrUMMS: \\ A New View of the Molecular Milky Way
}

\author{
Peter Barnes $^{1,2}$, Erik Muller ${ }^{3}$, Quang Nguyen Luong ${ }^{3}$, Hans Nguyen ${ }^{4}$ \\ ${ }^{1}$ Astronomy Dept., University of Florida, PO Box 112055, Gainesville FL 32611, USA \\ ${ }^{2}$ School of Science \& Technology, University of New England, Armidale NSW 2351, Australia \\ ${ }^{3}$ National Astronomical Observatory of Japan, Chile Observatory, Tokyo 181-8588, Japan \\ ${ }^{4}$ Max-Planck-Institut für Radioastronomie, Auf dem Hügel 69, 53121, Bonn, Germany \\ email: pjb@ufl.edu, erik.muller@nao.ac.jp, quang.nguyen-luong@nao.ac.jp, \\ hnguyen@cita.utoronto.ca
}

\begin{abstract}
We present the Three-mm Ultimate Mopra Milky Way Survey, a new mm-wave molecular-line mapping survey of the southern Galactic Plane, and its first data releases and science results. ThrUMMS maps a $60^{\circ} \times 2^{\circ}$ sector of our Galaxy's fourth quadrant, using a combination of fast mapping techniques with the Mopra radio telescope, simultaneously in the $J=1 \rightarrow 0$ lines of ${ }^{12} \mathrm{CO},{ }^{13} \mathrm{CO}, \mathrm{C}^{18} \mathrm{O}$, and $\mathrm{CN}$ near $112 \mathrm{GHz}$, at $1^{\prime} .2$ and $0.3 \mathrm{~km} \mathrm{~s}^{-1}$ resolution, with $1.2 \mathrm{~K} / \mathrm{ch}$ sensitivity for ${ }^{12} \mathrm{CO}$ and $0.7 \mathrm{~K} / \mathrm{ch}$ for the other transitions. The calibrated data cubes from these observations are made freely available to the community on the ThrUMMS website, http://www.astro.ufl.edu/thrumms, after processing through our pipeline. Here, we summarise the first science results, on global variations in the iso-CO line ratios and on a detailed multiwavelength study of the GMCs near $l=333^{\circ}$.
\end{abstract}

Keywords. Molecular clouds, Galactic structure, star formation

\section{Line Ratios and Molecular Mass}

In Barnes et al. (2015, Paper I), we found that the iso-CO line ratios vary dramatically across the Galactic Plane, indicating a very wide range of optical depth and excitation conditions, from warm and translucent to cold and opaque. Based on a simple yet robust radiative transfer analysis, including an innovative CO line ratio "colour-colour" diagram over all $10^{9}$ voxels, we showed that the population of cold clouds in particular have optical depths for ${ }^{12} \mathrm{CO}$ easily exceeding 100 in some locations. We also found wide variations in the intrinsic abundance ratio $R=\left[{ }^{13} \mathrm{CO}\right] /\left[\mathrm{C}^{18} \mathrm{O}\right]$. The high $\tau$ suggests that

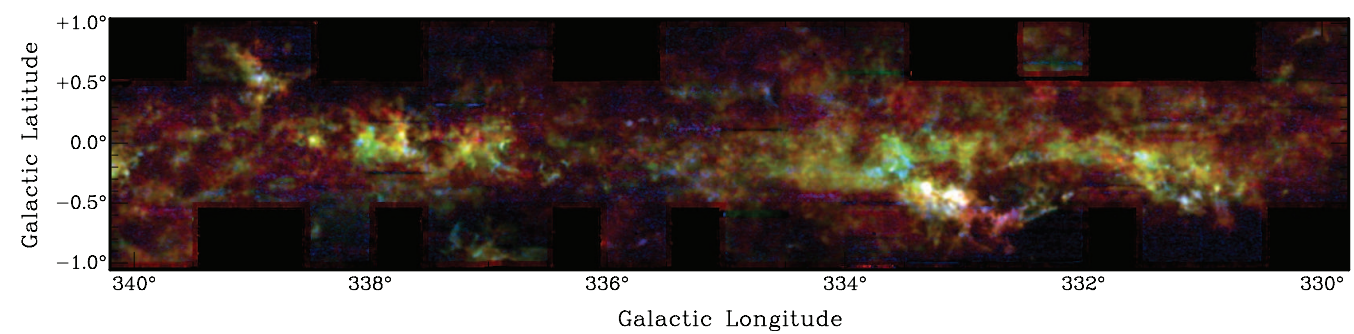

Figure 1. Sample colour composite map of ${ }^{12} \mathrm{CO}$ (red), ${ }^{13} \mathrm{CO}$ (green), and $\mathrm{C}^{18} \mathrm{O}$ (blue) integrated intensity images from DR3 of the ThrUMMS data cubes. Note the strong colour variations in both images, indicative of different line ratio, opacity, and excitation environments in the molecular material of the Milky Way. ThrUMMS provides similar data covering $360^{\circ}>l>300^{\circ}$ in all 3 iso-CO lines, plus CN. DR4, which includes improved spatial coverage and more uniform sensitivity, is now available. 


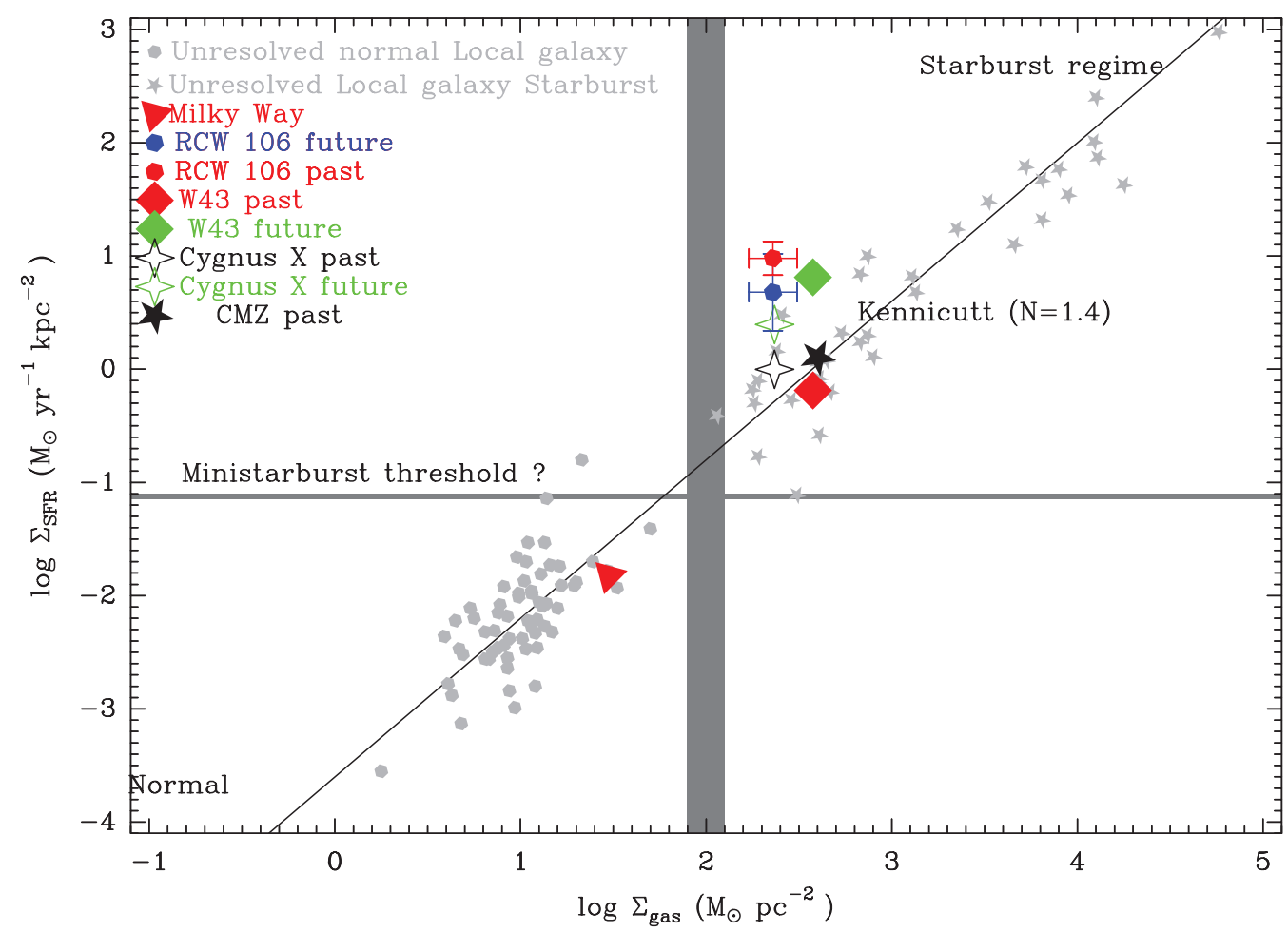

Figure 2. Schmidt Kennicutt relation between star formation rate (SFR) density and gas surface density, from normal spiral galaxies to starbursts. Points for the RCW 106 complex and other Milky Way locations suggest that RCW 106 is the most vigorous star formation complex in the entire Galaxy.

the fraction of the molecular mass in the Galactic disk that is in the coldest gas may be substantially underestimated. We computed column densities from the global data and derived a new conversion law from $\mathrm{CO}$ to molecular mass,

$$
N(\mathrm{CO})=1.6 \times 10^{20} \mathrm{~mol} \mathrm{~m}^{-2}\left(I_{\mathrm{CO}} / \mathrm{K} \mathrm{km} \mathrm{s}^{-1}\right)^{1.38},
$$

which typically gives mass columns $2-3 \times$ larger than given by the standard "X-factor" approach. This means that global relationships in disk galaxies that depend on the $\mathrm{CO} \rightarrow \mathrm{H}_{2}$ mass conversion, such as star formation laws, may need to be recalibrated.

\section{The RCW 106 Ministarburst}

Near $l=333^{\circ}$, we compared ThrUMMS data to HI, cm-continuum, and several Herschel and Spitzer bands to derive the overall mass and star formation properties of two complexes along the line of sight, in the Scutum-Centaurus and Norma arms. The RCW 106 complex in particular is currently undergoing a ministarburst event, potentially shedding light on starburst physics in distant galaxies.

\section{References}

Barnes, P.J., Muller, E., Indermuehle, B., et al. 2015, ApJ, 812, 6

Nguyen, H., Nguyen Luong, Q., Martin, P.G., et al. 2015, ApJ, 812, 7 